\title{
QCD Issues in Searches for Supersymmetry with the ATLAS Detector*
}

\author{
A. Santra (on behalf of the ATLAS Collaboration) ${ }^{\mathrm{a}, 1}$ \\ ${ }^{a}$ Instituto de Física Corpuscular, University of Valencia / CSIC, Valencia, C/Catedrátic José Beltrán Martinez, 2, 46980 Paterna, Valencia, Spain
}

\begin{abstract}
Even though the experimental evidence is absent, weak-scale supersymmetry (SUSY) remains one of the best motivated and studied Standard Model extensions to date. There are many ways that searches for weak-scale supersymmetry depend on QCD: QCD scaling rules are used in background estimation techniques, jet sub-structure is exploited for separating signal and background, signal cross-sections rely on higher-order calculations and resummation, etc. For the search for R-hadrons, hadrons including long-lived SUSY particles, additional complex QCD issues arise. This proceeding summarizes recent ATLAS results on supersymmetry searches, with a focus on those aspects that most strongly depend on QCD.
\end{abstract}

Keywords: SUSY, QCD, jet sub-structure, R-Hadrons

\section{Introduction}

The Standard Model (SM) of particle physics is the most successful theory for elementary particles at present. Yet there are many aspects, for example, hierarchy problem or the nature of the dark matter, which SM cannot explain. Supersymmetry (SUSY) [1-6] is, at present, one of the most popular beyond-SM (BSM) theories. SUSY postulates that there exists a fundamental symmetry of spacetime which relates all the SM particles to their supersymmetric partners with spins shifted by $1 / 2$.

Multipurpose detectors like ATLAS [7] and CMS [8] search extensively for SUSY at the LHC. Numerous searches for SUSY in a wide variety of final states have been conducted by these experiments. In this proceeding, searches for a few SUSY scenarios in the ATLAS experiment will be discussed. In these searches, QCD

*Talk given at 22nd High Energy Physics International Conference In Quantum Chromodynamics (QCD 19), 2 July - 5 July, 2019, Montpellier - FR

Email address: arka.santra@cern. ch (A. Santra (on behalf of the ATLAS Collaboration))

${ }^{1}$ (C) 2019 CERN for the benefit of the ATLAS Collaboration. CCBY-4.0 license. effects play a key role. For example, QCD scaling rules are applied in background estimation techniques for some searches, while jet sub-structure is exploited as a discriminating variable between signal and background. Additional complications arise for the search for R-hadrons, which include long-lived SUSY particles.

The datasets discussed in this proceeding were collected from $p p$ collisions at $\sqrt{s}=13 \mathrm{TeV}$, with integrated luminosity varying from $3.2 \mathrm{fb}^{-1}$ to $36.1 \mathrm{fb}^{-1}$.

\section{Lepton and high jet multiplicity final state search}

A large number of SUSY searches have been carried out by the ATLAS experiment, but there was a gap in the search coverage where the final state contains one or more leptons, many jets and little or no missing transverse energy $\left(E_{\mathrm{T}}^{\mathrm{miss}}\right)$. This gap was filled by one ATLAS search [9] that targeted R-parity violating (RPV) SUSY models.

For this analysis, the dominant sources of background come from the top-quark pair production $(t \bar{t}+$ jets $)$ and $W / Z+$ jets production. Since simulating these kind of events suffers from high theoretical uncertainties at high jet multiplicities, the backgrounds were estimated from 
the data-driven technique. Here the $b$-tagged jet multiplicity distribution obtained from the moderate jet multiplicities was extrapolated to the high jet multiplicities of the search region. The different sources of background for different numbers of $b$-tagged jets for this analysis are shown in Figure 1.

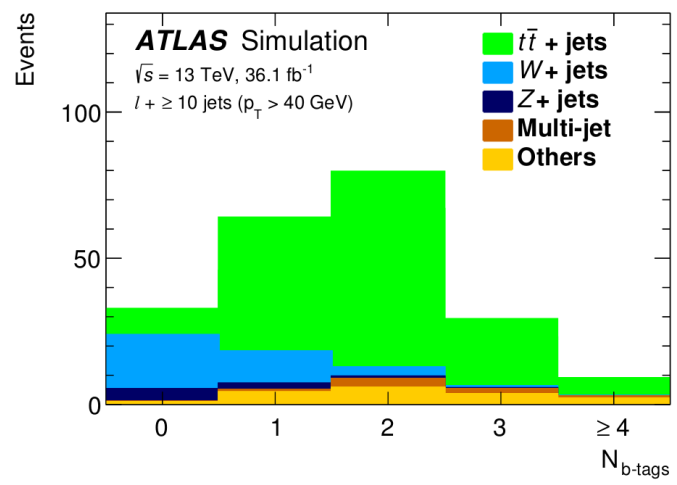

Figure 1: The expected background from simulation in the different $b$-tagged jet bin. The events have at least ten jets with $p_{\mathrm{T}}>40 \mathrm{GeV}$ [9].

To determine the $W / Z+$ jets background, a partially data-driven approach is adopted where the shape of the $b$-tag multiplicity distribution is taken from simulation, while the normalization in each jet slice is taken from data. The estimate of the normalization relies on assuming a functional form which describes the evolution of the number of $W / Z+$ jets with respect to the jet multiplicity, $r(j) \equiv N_{\mathrm{j}+1}^{W / Z+\mathrm{jets}} / N_{\mathrm{j}}^{W / Z+\mathrm{jets}}$. The function $r(j)$ can be parameterized as:

$$
r(j)=c_{0}+c_{1} /(j+1)
$$

where $c_{0}$ and $c_{1}$ constants are extracted from the fit to the data. Figure 2, where each panel shows the ratio for data/MC simulation with the fitted parameterization, validates the jet scaling parameterization of the ratio $r(j)$ [9].

To determine the $t \bar{t}+$ jets background, an initial template of the $b$-tag multiplicity distribution was made in events with five jets. The evolution of this template to higher jet multiplicities was parameterized, and the parameters were determined from the fit to the data [9].

In this analysis, no significant excess over the expected background was found in data and the results are interpreted in the framework of simplified models featuring gluino or top squark pair production in RPV SUSY scenarios (Figure 3).

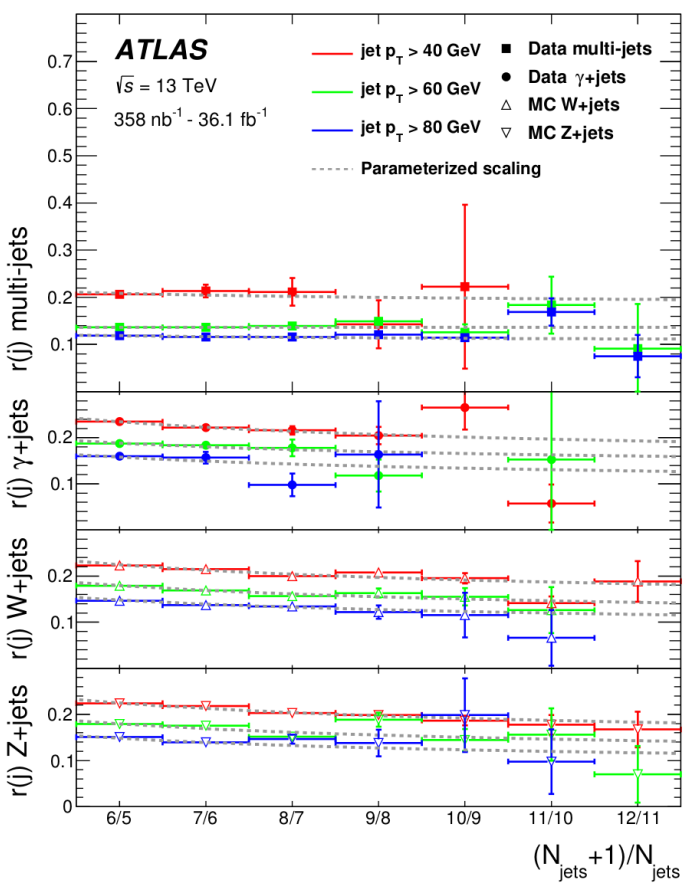

Figure 2: The ratio $(r(j))$ of number of events with $(j+1)$ jets to the number of events with $j$ jets for various processes. These ratios are used to validate the jet-scaling parameterization [9].

\section{Search for R-parity violating SUSY particles in multi-jet final state}

One ATLAS analysis [10] searched for supersymmetric gluino pair production with subsequent R-parity violating [11-16] decays, which leads to many jets (at least four or five jets depending on the signal region) in the final state. This analysis searched for scenarios where baryon-number-violating coupling $\left(\lambda^{\prime \prime}\right)$ were taken to be non-zero.

The dominant background comes from the SM multijet processes. This analysis uses a kinematic variable called total jet mass $\left(M_{J}^{\Sigma}\right)$ [17-19] as a primary discriminating variable between signal and background. The $M_{J}^{\Sigma}$ is defined as the sum of the masses of the four leading large- $\mathrm{R}$ jets (jets constructed with the radius parameter $\mathrm{R}=1.0$ ) having $p_{\mathrm{T}}>200 \mathrm{GeV}$ and $|\eta| \leq 2.0$. The plot in Figure 4 (top) shows the example of the discrimination the $M_{J}^{\Sigma}$ variable provides.

Apart from the $M_{J}^{\Sigma}$, another discriminating variable used in this analysis was $\left|\Delta \eta_{12}\right|$, the pseudo-rapidity difference between the two leading large- $\mathrm{R}$ jets. The distribution variable is shown in Figure 4 (bottom).

The use of the $M_{J}^{\Sigma}$ variable allows an opportunity to use the fully data-driven jet mass template method [20] 

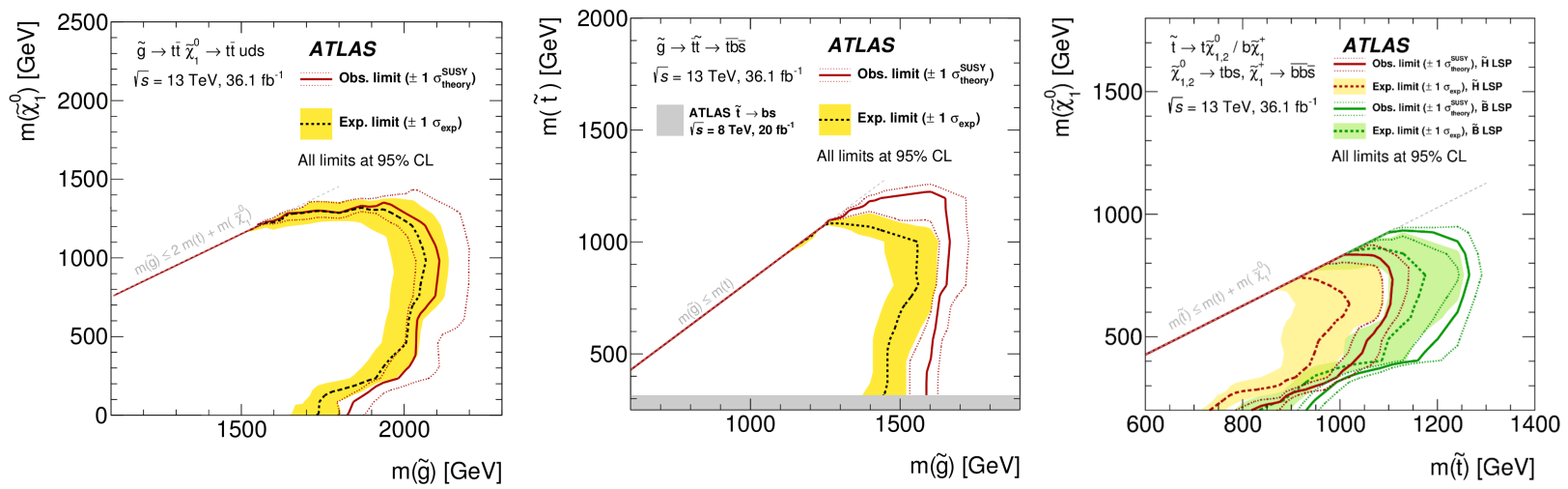

Figure 3: The 95\% CL exclusion contours as a function of gluino $(\tilde{g})$ and neutralino $\left(\tilde{\chi}_{1}^{0}\right)$ mass (left), gluino and stop ( $\left.\tilde{t}\right)$ mass (middle) and stop and neutralino mass (right) in the context of the RPV SUSY scenarios probed in the analysis [9].

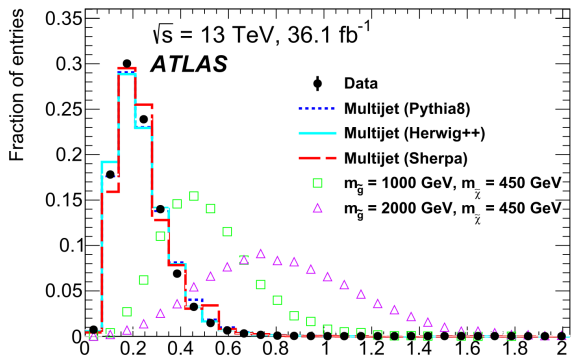

$M_{\mathrm{J}}^{\Sigma}[\mathrm{TeV}]$

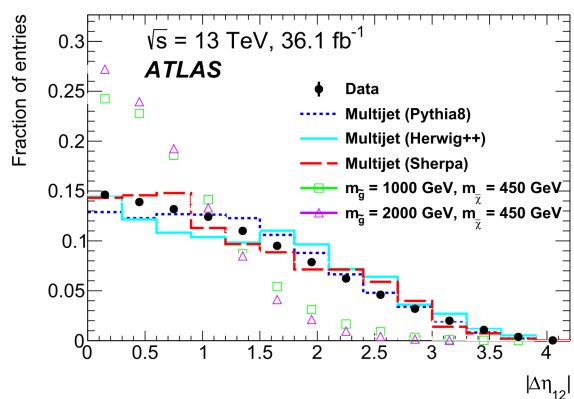

Figure 4: Comparison between signal and background control samples for the total jet mass defined in the text (top) and the difference in pseudo-rapidity between two leading large- $R$ jets (bottom). These variables can be used as discriminating variables between signal and background [10]

to estimate the background contributions in the signal region. Here single jet mass templates are extracted from control regions devoid of signal contamination. These templates are made in bins defined by a number of observables, including jet $p_{\mathrm{T}}$ and $|\eta|$, and the $b$ matching status. These templates provide a probability density function describing the relative probability of a jet with a certain $p_{\mathrm{T}}$ and $|\eta|$ to have a certain mass. For each jet in the validation region or signal region, its cor- responding jet mass template is used to generate a random jet mass, which is further used to construct the $M_{J}^{\Sigma}$ distribution in that region. The distribution of $M_{J}^{\Sigma}$ from background sources and the observed data is shown in Figure 5 for a signal region having at least four jets. Since there was no significant excess in observed data over the predicted background found in this analysis, this analysis sets limits on relevant SUSY parameters (Figure 6).

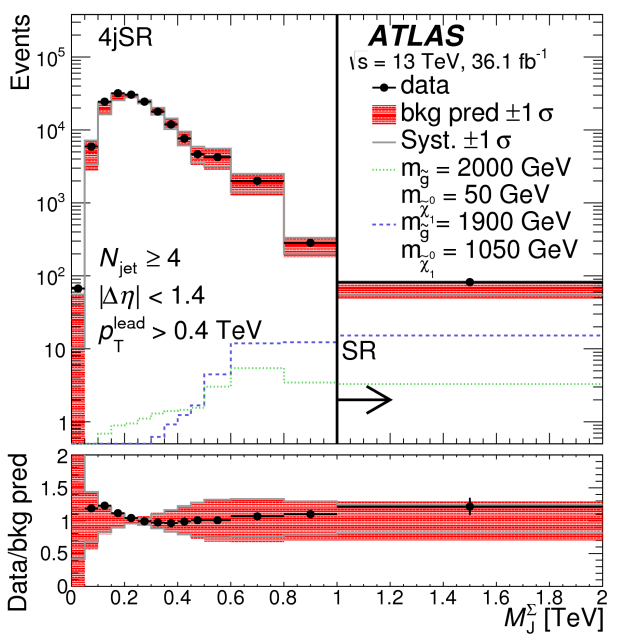

Figure 5: The observed (black dots) and the predicted (red solid line) $M_{J}^{\Sigma}$ distribution for the signal region with at least four jets [10].

\section{Search for SUSY with large jet multiplicities and missing transverse energy}

The ATLAS experiment searched for SUSY with at least seven jets and large missing transverse energy in 


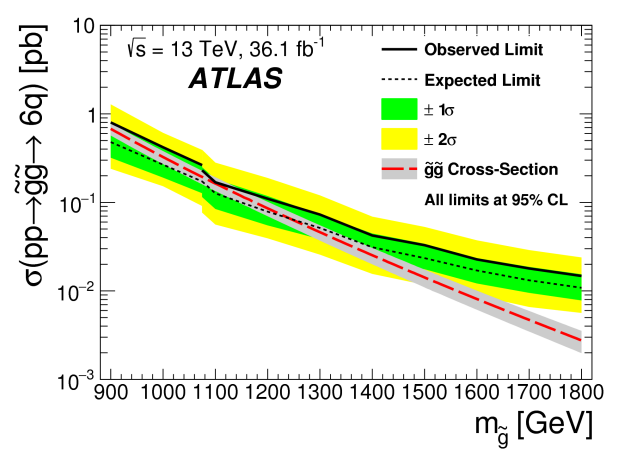

Figure 6: Observed and expected cross-section limits for the gluino direct decay model [10].

the collision data collected during 2015 and 2016 [21]. The selected events were further classified into events containing $b$-tagged jets or on the sum of large-radius jets. The dominant background from SM multi-jet processes was determined using a data-driven technique. Other significant background contributions came from top quark pair production and $W+$ jets samples.

The target signal events for this analysis have a large number of jets, beyond what is generated by SM processes. Those events also have high $E_{\mathrm{T}}^{\text {miss }}$ which is significantly larger than that expected from detector-related effects. Signal regions require high value of the ratio $E_{\mathrm{T}}^{\mathrm{miss}} / H_{\mathrm{T}}$ where $H_{\mathrm{T}}$ is defined as the scalar sum of jet transverse momenta (jets being restricted to have $p_{\mathrm{T}}>40 \mathrm{GeV}$ and $\left.|\eta|<2.8\right)$.

This analysis observed that the $E_{\mathrm{T}}^{\text {miss }} / H_{\mathrm{T}}$ spectrum for selected multi-jet events is approximately independent of how $H_{\mathrm{T}}$ is partitioned between the jets present in the events. A template for the multi-jet $E_{\mathrm{T}}^{\text {miss }} / H_{\mathrm{T}}$ distribution was constructed from a selection complementary to the signal region. While using the template in the signal region, care was taken to estimate the effect of jet flavor composition, pile-up and the division of $H_{\mathrm{T}}$ among the multiple jets in a single event [21].

Leptonic backgrounds ( $W \rightarrow l v$ or $Z \rightarrow v v$ decays, single top or $t \bar{t}$ etc) constituted a small background, and they were estimated from the simulation.

The observed data and the expected background for all signal regions considered in this analysis is shown in Figure 7. As the observed data were not significantly higher than the expected background, this analysis produced $95 \%$ CL limits on the masses of gluino and neutralino (Figure 8).

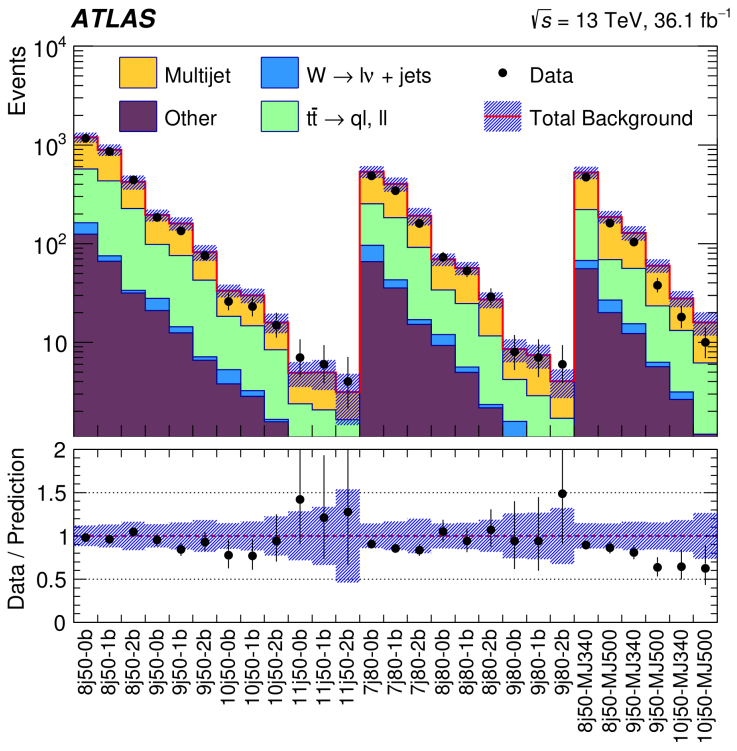

Figure 7: Data observed and expected background for all the signal regions considered in the analysis [21].

\section{Search for ling-lived charged R-hadrons}

R-hadrons are composite colorless states of squarks or gluinos together with SM quarks or gluons. The search for R-hadrons [22] exploits the fact these particles are expected to travel with velocity $(\beta=v / c)$ much slower than one and to exhibit a specific ionization energy loss $(d E / d x)$ larger than that for any charged SM particle.

To determine $\beta$, the time of flight measurement is needed. This measurement relies on the timing and distance information from tile calorimeter cells, which are crossed by the extrapolated candidate tracks in three radial layers in the central barrel and an extended barrel on each side. Figure 9 shows the resolution of $\beta$ measurement in the ATLAS tile calorimeter cells. The final $\beta$ measurement is done by a weighted average of the $\beta$ measurements in the cells crossed by the candidate particles and after the application of a series of calibration techniques [22]. Cells which have energy less than $E_{\text {min,cell }}=500 \mathrm{MeV}$ are not taken into account. This is done to reduce the effect of detector noise. After all calibrations are applied, the single cell time resolution ranges from $1.3 \mathrm{~ns}$ (large radii) to $2.5 \mathrm{~ns}$ (small radii).

The expected $\beta$ distribution for the background is determined from data, but for the SUSY signal samples one has to rely on the simulated distribution of $\beta$. The simulation is validated by comparing the $\beta$ distribution of $Z \rightarrow \mu \mu$ from data and simulation.

To estimate the background for this analysis, a proba- 

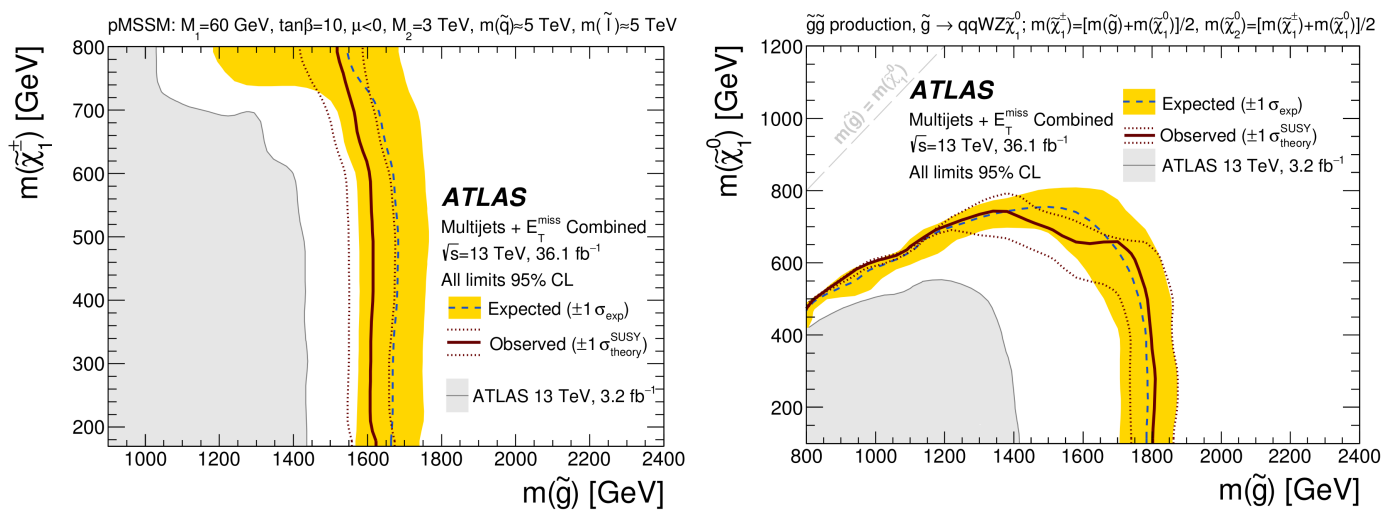

Figure 8: Exclusion contours in the gluino and chargino mass plane for the pMSSM model (left) and gluino and neutralino mass plane for the simplified model with two-step cascade decay of gluinos (right) [21].

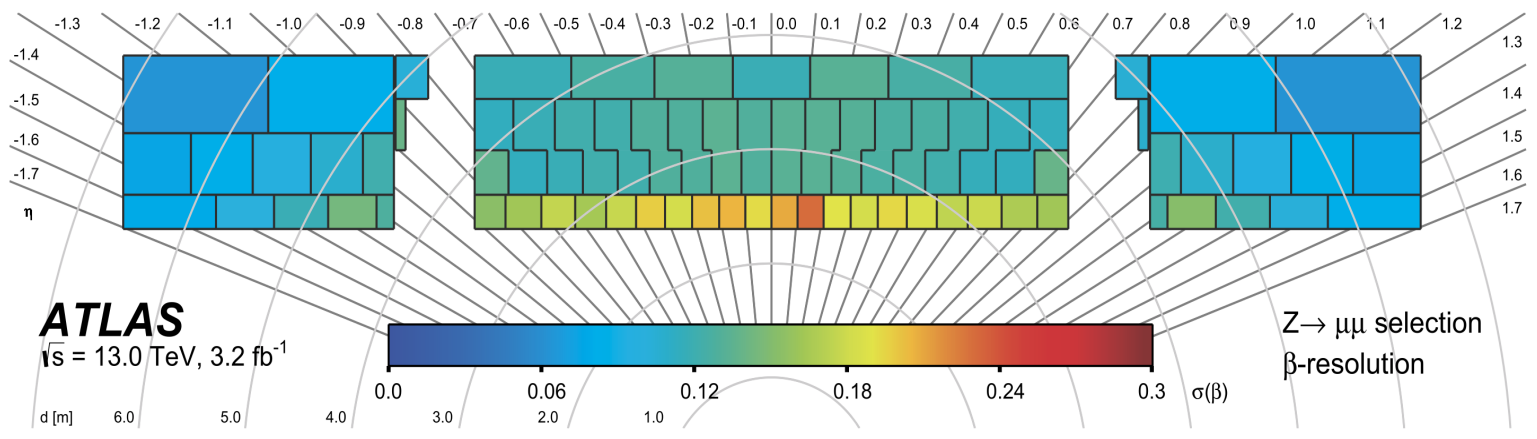

Figure 9: Resolution of $\beta$ for different tile calorimeter cells of ATLAS. This is obtained from a $Z \rightarrow \mu \mu$ selection in data [22].

bility distribution function in the momentum $(p), \beta$ and $\beta \gamma$ values are determined from the sideband of the signal region. Here $\beta$ refers to the time-of-flight-based measurements in the tile calorimeter and $\beta \gamma$ refers to the quantities derived from the $d E / d x$ measurement in the silicon pixel detector. The $m_{\beta}$ distribution from data and background, where $m=p / \beta \gamma$ was used to get $m_{\beta}$, are shown in Figure 10.

Only two events passed the event selection for the Rhadron with $1000 \mathrm{GeV}$ mass hypothesis, where the expected background was $1.23 \pm 0.37$. Since there was no significant excess in data over the background in the signal region, this analysis set 95\% CL upper limit on the production cross-section, as shown in Figure 11.

\section{Conclusion}

The ATLAS experiment is pursuing an extensive search program for SUSY at the electroweak scale. For many searches where QCD is playing an important role, different techniques (mostly data-driven) are adopted to estimate the QCD background. Signal cross-sections rely on higher order QCD loop calculations and resummation. As good agreement is found between observed data and expected background, the ATLAS experiment is putting limits on the production cross-sections and relevant SUSY particle masses.

Future searches at the ATLAS detector plan to use full dataset obtained during LHC Run 2 with integrated luminosity of about $140 \mathrm{fb}^{-1}$. There are many software and reconstruction $\mathrm{R} \& \mathrm{D}$ programs also going on to improve the search result. These programs will help to increase the reach of SUSY searches to new and more difficult parameter spaces and to test many new wellmotivated physics scenarios.

\section{Acknowledgement}

The author acknowledges the support by the Generalitat Valenciana through a MoEDAL-supporting agreement, by the grant PGC2018-094856-B-I00 (MCIU/AEI/FEDER, UE) and by the Severo Ochoa Excellence Centre Project SEV-2014-0398. 


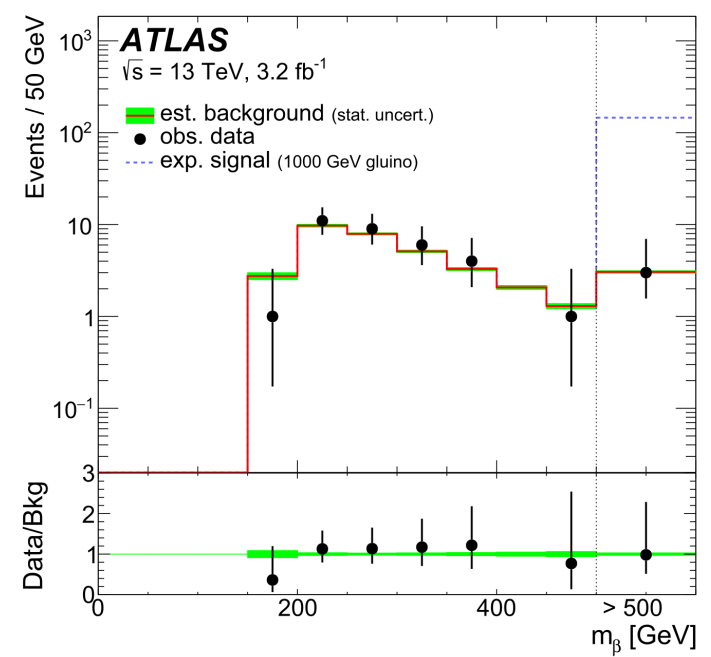

Figure 10: Data (in black dots) and estimated background (in red line) for $m_{\beta}$ for the gluino R-hadron search (mass $1000 \mathrm{GeV}$ ). The dashed blue lines show the expected signal on top of the background, and the green band shows the statistical uncertainty of the background estimate [22].

\section{References}

[1] Yu. A. Golfand and E. P. Likhtman. Extension of the Algebra of Poincare Group Generators and Violation of $p$ Invariance. JETP Lett., 13:323, 1971. [Pisma Zh. Eksp. Teor. Fiz. 13 (1971) 452]. URL: http://inspirehep.net/record/68412.

[2] D. V. Volkov and V. P. Akulov. Is the Neutrino a Goldstone Particle? Phys. Lett. B, 46:109, 1973. doi:10.1016/03702693(73)90490-5.

[3] J. Wess and B. Zumino. Supergauge Transformations in FourDimensions. Nucl. Phys. B, 70:39, 1974. doi:10.1016/05503213(74)90355-1.

[4] J. Wess and B. Zumino. Supergauge Invariant Extension of Quantum Electrodynamics. Nucl. Phys. B, 78:1, 1974. doi:10.1016/0550-3213(74)90112-6.

[5] S. Ferrara and B. Zumino. Supergauge Invariant Yang-Mills Theories. Nucl. Phys. B, 79:413, 1974. doi:10.1016/05503213(74)90559-8.

[6] Abdus Salam and J. A. Strathdee. Supersymmetry and Nonabelian Gauges. Phys. Lett. B, 51:353, 1974. doi:10.1016/0370-2693(74)90226-3.

[7] ATLAS Collaboration, Journal of Instrumentation 3, S08003 (2008). doi:10.1088/1748-0221/3/08/s08003

[8] CMS Collaboration, JINST 3, S08004 (2008). doi:10.1088/1748-0221/3/08/S08004

[9] ATLAS collaboration, J. High Energ. Phys. (2017) 88. https://doi.org/10.1007/JHEP09(2017)088.

[10] ATLAS collaboration, Physics Letters B (2018) 785:136-158. https://doi.org/10.1016/j.physletb.2018.08.021.

[11] G.R. Farrar, P. Fayet, Phenomenology of the production, decay, and detection of new hadronic states associated with supersymmetry, Phys. Lett. B 76 (1978) 575.

[12] L.J. Hall, M. Suzuki, Explicit R-parity breaking in supersymmetric models, Nucl. Phys. B 231 (1984) 419.

[13] G.G. Ross, J.W.F. Valle, Supersymmetric models without Rparity, Phys. Lett. B 151 (1985) 375.

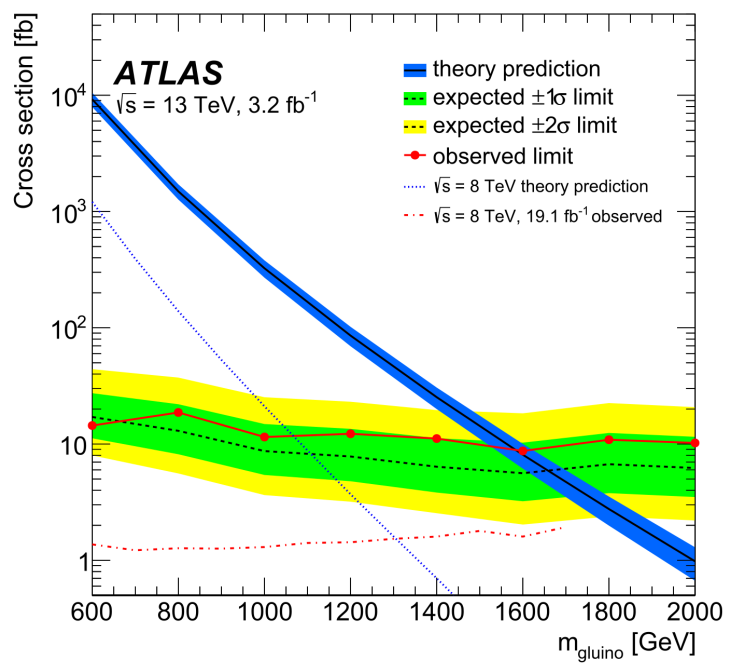

Figure 11: 95\% CL upper limits on the cross-section as a function of mass for the production of long-lived gluino R-hadrons. The dashed black line shows the expected upper limits, and the solid red line shows the observed upper limits [22].

[14] V.D. Barger, G.F. Giudice, T. Han, Some new aspects of supersymmetry R-parity violating interactions, Phys. Rev. D 40 (1989) 2987.

[15] H.K. Dreiner, An introduction to explicit R-parity violation, Adv. Ser. Dir. High Energy Phys. 21 (1997) 565, arXiv:hep -ph /9707435.

[16] R. Barbier, et al., R-parity violating supersymmetry, Phys. Rep. 420 (2005) 1, arXiv:hep-ph /0406039.

[17] A. Hook, E. Izaguirre, M. Lisanti, J.G. Wacker, High multiplicity searches at the LHC using jet masses, Phys. Rev. D 85 (2012) 055029, arXiv:1202.0558 [hep-ph].

[18] S. El Hedri, A. Hook, M. Jankowiak, J.G. Wacker, Learning how to count: a high multiplicity search for the LHC, J. High Energy Phys. 08 (2013) 136, arXiv:1302.1870 [hep -ph].

[19] T. Cohen, M. Jankowiak, M. Lisanti, H.K. Lou, J.G. Wacker, Jet substructure tem- plates: data-driven QCD backgrounds for fat jet searches, J. High Energy Phys. 05 (2014) 005, arXiv:1402.0516 [hep -ph].

[20] T. Cohen, M. Jankowiak, M. Lisanti, H.K. Lou, J.G. Wacker, Jet substructure tem- plates: data-driven QCD backgrounds for fat jet searches, J. High Energy Phys. 05 (2014) 005, arXiv: 1402.0516 [hep -ph].

[21] ATLAS collaboration, J. High Energ. Phys. (2017) 34. https://doi.org/10.1007/JHEP12(2017)034

[22] ATLAS collaboration, Physics Letters B (2016) 760:647-665. https://doi.org/10.1016/j.physletb.2016.07.042 